\title{
A distribuição Weibull Exponencial de Zografos
}

\author{
Patrícia Silva N. Barros, \\ PPGBEA - UFRPE / DCE - UFPB \\ E-mail: patriciajp1408@hotmail.com \\ Kleber Napoleão N. de O. Barros , \\ PPGBEA - UFRPE / DE - UEPB \\ E-mail: kleber.barros@cct.uepb.br \\ Cícero Carlos R. de Brito, Wilson Rosa de Oliveira Junior \\ PPGBEA - UFRPE \\ E-mail: cicerocarlosbrito@yahoo.com.br, wilson.rosa@gmail.com
}

\begin{abstract}
Resumo: A distribuição Weibull é muito popular para dados em modelagem de vida. Em [3] é apresentada uma introdução a família gama-generalizada de distribuições. Daí tem-se, como proposta, a distribuição Weibull Exponencial de Zografos (WEZ). A WEZ tem a distribuição Weibull, exponencial e Rayleigh como casos particulares. Um tratamento compreensivo das propriedades matemáticas da WEZ é feita, sendo encontradas as expressões para a média, variância, coeficiente de assimetria e curtose e fez-se um ajuste pelo método da máxima verossimilhança. Verificando pela log-verossimilhança que o melhor ajuste foi das estimativas pelo método de máxima verossimilhança. Dessa forma a distribuição Weibull Exponencial de Zografos pode ser utilizada para ajustar dados de análise de sobrevivência.
\end{abstract}

Palavras-chave: Distribuição Weibull, Família gama-generalizada, Distribuição Weibull Exponencial de Zografos

\section{Introdução}

A distribuição Weibull, tendo exponencial e Rayleigh como casos especiais, é uma distribuição muito popular para dados em modelagem de vida. Nos últimos anos, novas classes de distribuições foram propostas com base em modificações da distribuição Weibull para lidar com a taxa de falha em forma de banheira. Uma boa revisão de alguns destes modelos é apresentada por [1].

O artigo [2], propôs a distribuição Beta Weibull Modificada (BWM) que apresentou flexibilidade para acomodar várias formas da função de taxa de falha, por exemplo, forma de U e unimodal, sendo, portanto, muito utilizada em uma variedade de aplicações nas áreas de confiabilidade, Medicina e Biologia, bem como em outras áreas de investigação.

Este artigo tem o objetivo de utilizar a generalização proposta por [3] para generalizar a distribuição Weibull. Pretende-se desenvolver a função densidade de probabilidade, a função de risco, a média, a variância, o coeficiente de assimetria, o coeficiente de curtose e o ajuste pelo método de máxima verossimilhança da nova distribuição: Weibull Exponencial de Zografos.

\section{Metodologia}

A distribuição de Weibull foi proposta originalmente por W. Weibull em estudos relacionados ao tempo de falha devido a fadiga de metais. A distribuição Weibull é uma distribuição proposta para análise de confiabilidade. Ela é uma distribuição muito flexível, e por esta razão, amplamente utilizada. Na maioria dos casos, a função densidade é definida como [4] 


$$
f(t)=\theta \lambda^{\theta} t^{\theta-1} e^{-(\lambda t)^{\theta}}, 0<t<\infty
$$

Foram desenvolvidas e discutidas várias formas de generalização de distribuições nos últimos anos. Foi introduzida em [3] a família gama-generalizada de distribuições. Definiram a função de distribuição acumulada da gama-generalizada (para $x \in R$ ) por:

$$
F(x)=\frac{1}{\Gamma(\delta)} \int_{0}^{-\log [\bar{G}(x ; \xi)]} t^{\delta-1} e^{-t} d t
$$

em que $\bar{G}(x)=1-G(x)$ é a função de sobrevivência e $\Gamma(\delta)=\int_{0}^{\infty} x^{\delta-1} e^{-x} d x$ é a função gama.

Neste artigo utilizamos esta forma de generalização para a distribuição Weibull. Desta forma a função de distribuição acumulada da distribuição Weibull Exponencial de Zografos é dada por:

$$
\begin{gathered}
F(x)=\int_{0}^{-\log \left(e^{-\beta x}\right)} \lambda \theta(\lambda t)^{\theta-1} e^{-(\lambda t)^{\theta}} d t \\
F(x)=1-e^{-(\beta \lambda x)^{\theta}}
\end{gathered}
$$

em que $x>0, \beta>0, \theta>0$ e $\lambda>0$. Neste caso utilizou-se $\bar{G}(x)=e^{-\beta x}$ que é a função de sobrevivência da Exponencial. Os parâmetros $\beta>0$ e $\theta>0$ são de forma, $\lambda>0$ é o de escala. A função densidade é dada por:

$$
f(x)=\beta \theta \lambda(\lambda \beta x)^{\theta-1} e^{-(\beta \lambda x)^{\theta}}
$$

em que $x>0, \beta>0, \theta>0$ e $\lambda>0$.

A distribuição Weibull Exponencial de Zografos tem como casos especiais: para $\beta=1$ tem-se a distribuição Weibull; para $\beta=1$ e $\theta=1$ tem-se a distribuição exponencial; e para $\beta=1$, $\theta=2$ e $\lambda=1 / \sigma$ tem-se a distribuição Rayleigh.

A função de risco é usada para calcular o risco de um dado indivíduo vir a falhar em um dado instante t e é obtida pela seguinte relação:

$$
h(t)=\frac{f(t)}{S(t)}
$$

Substituindo $f(t)$ e $S(t)=1-F(t)$ na expressão anterior pela função densidade de probabilidade e a função de sobrevivência da distribuição Weibull Exponencial de Zografos, a função de risco é dada por:

$$
h(x)=\beta \theta \lambda(\lambda \beta x)^{\theta-1}
$$

em que $x>0, \beta>0, \theta>0$ e $\lambda>0$.

É necessário enfatizar a importância e a necessidade dos momentos em qualquer análise estatística especialmente em trabalhos aplicados. Algumas das mais importantes características de uma dada distribuição pode ser estudada através dos momentos: média, variância, coeficiente de assimetria e curtose. Seja $X$ uma variável aleatória com função densidade de probabilidade dada pela distribuição Weibull Exponencial de Zografos os quatro primeiros momentos, a média, a variância, o coeficiente de assimetria e o coeficiente de curtose são dados, respectivamente, por:

$$
\begin{gathered}
\mu=\frac{\Gamma\left[\frac{1}{\theta}\right]}{\beta \lambda \theta} \\
\operatorname{Var}=\frac{\theta^{2} \Gamma\left[\frac{2+\theta}{\theta}\right]-\Gamma\left(\frac{1}{\theta}\right)^{2}}{\beta^{2} \lambda^{2} \theta^{2}}
\end{gathered}
$$




$$
\begin{gathered}
\text { Assimetria }=\frac{\theta^{3} \Gamma\left[\frac{3+\theta}{\theta}\right]+2 \Gamma\left[\frac{1}{\theta}\right]^{3}-6 \theta \Gamma\left[\frac{1}{\theta}\right] \Gamma\left[\frac{2}{\theta}\right]}{\beta^{3} \lambda^{3} \theta^{3}\left(\frac{\theta^{2} \Gamma\left[\frac{2+\theta}{\theta}\right]-\Gamma\left[\frac{1}{\theta}\right]^{2}}{\beta^{2} \lambda^{2} \theta^{2}}\right)^{3 / 2}} \\
\text { Curtose }=\frac{\theta^{4} \Gamma\left[\frac{4+\theta}{\theta}\right]-3 \Gamma\left[\frac{1}{\theta}\right]\left(\Gamma\left[\frac{1}{\theta}\right]^{3}-4 \theta \Gamma\left(\frac{1}{\theta} \Gamma\left[\frac{2}{\theta}\right]+4 \theta^{2} \Gamma\left[\frac{3}{\theta}\right]\right)\right.}{\left(\theta^{2} \Gamma\left[\frac{2+\theta}{\theta}\right]-\Gamma\left[\frac{1}{\theta}\right]^{2}\right)^{2}}
\end{gathered}
$$

Sejam $X_{1}, X_{2}, \ldots, X_{n}$ variáveis aleatórias, independentes e identicamente distribuídas seguindo uma WEZ e $\boldsymbol{\xi}=(\beta, \lambda, \theta)^{\prime}$, a função de verossimilhança é expressa como segue:

$$
L(\boldsymbol{\xi})=(\beta \theta \lambda)^{n} \prod_{i=1}^{n}\left(\lambda \beta x_{i}\right)^{\theta-1} e^{-\sum_{i=1}^{n}(\beta \lambda x)^{\theta}}
$$

O logaritmo da função de verossimilhança é dada por:

$$
l(\boldsymbol{\xi})=\log (L(\boldsymbol{\xi}))=n \log (\beta \theta \lambda)+(\theta-1) \log \prod_{i=1}^{n}\left(\lambda \beta x_{i}\right)-\sum_{i=1}^{n}(\beta \lambda x)^{\theta}
$$

\section{Resultados e Discussões}

A seguir estão os gráficos da função densidade de probabilidade, a função de risco, a média, a variância, o coeficiente de assimetria e curtose e o ajuste pela função de verossimilhança da distribuição Weibull Exponencial de Zografos.

A Figura 1 ilustra algumas das formas da função densidade de probabilidade e da função de risco com $\lambda$ e $\theta$ fixos e variando o $\beta$. Verifica-se que a função densidade tem formas diferentes para cada $\beta$, quando $\beta$ tende a 1 se aproxima da Weibull. A função de risco é crescente para qualquer valor de $\beta$, porém quanto maior for o valor atribuído a este parâmetro, maior será o risco.
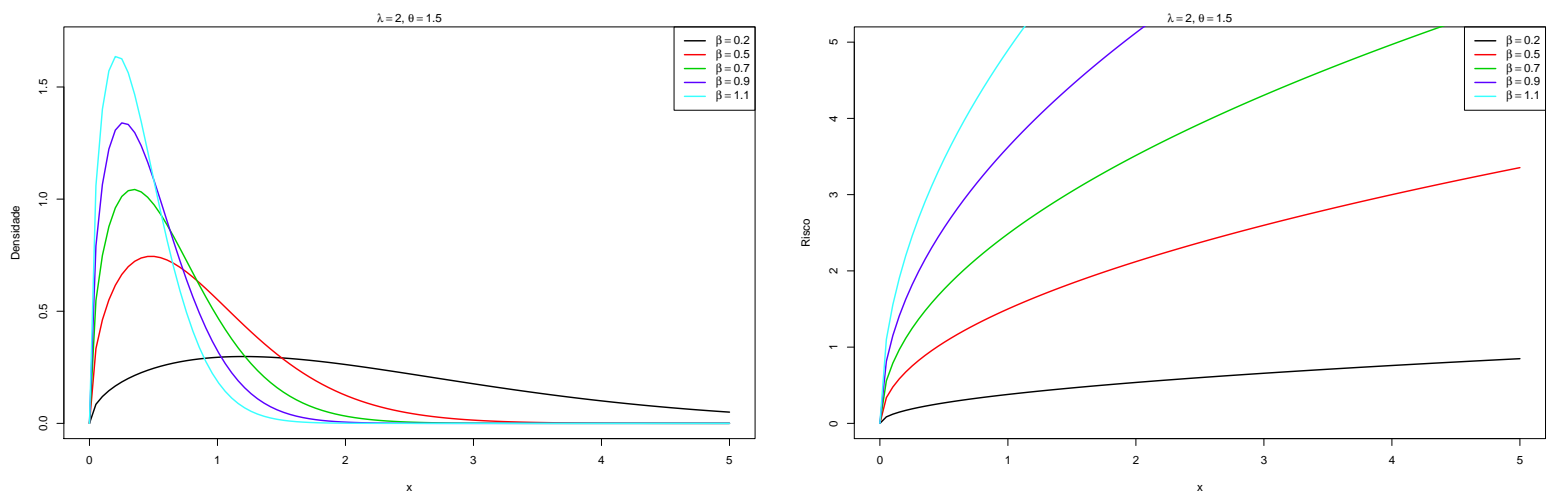

Figura 1: Função densidade de probabilidade e função de risco com $\lambda$ e $\theta$ fixos e variando o $\beta$

A Figura 2 apresenta a função densidade de probabilidade e a função de risco com $\beta$ e $\theta$ fixos e variando o $\lambda$. Observa-se que a função densidade tem a mesma forma diferenciando a escala, quando o $\lambda$ aumenta a dispersão diminui. O comportamento da função de risco para $\lambda$ fixo é semelhante ao ocorrido com $\beta$, porém com amplitude reduzida. 

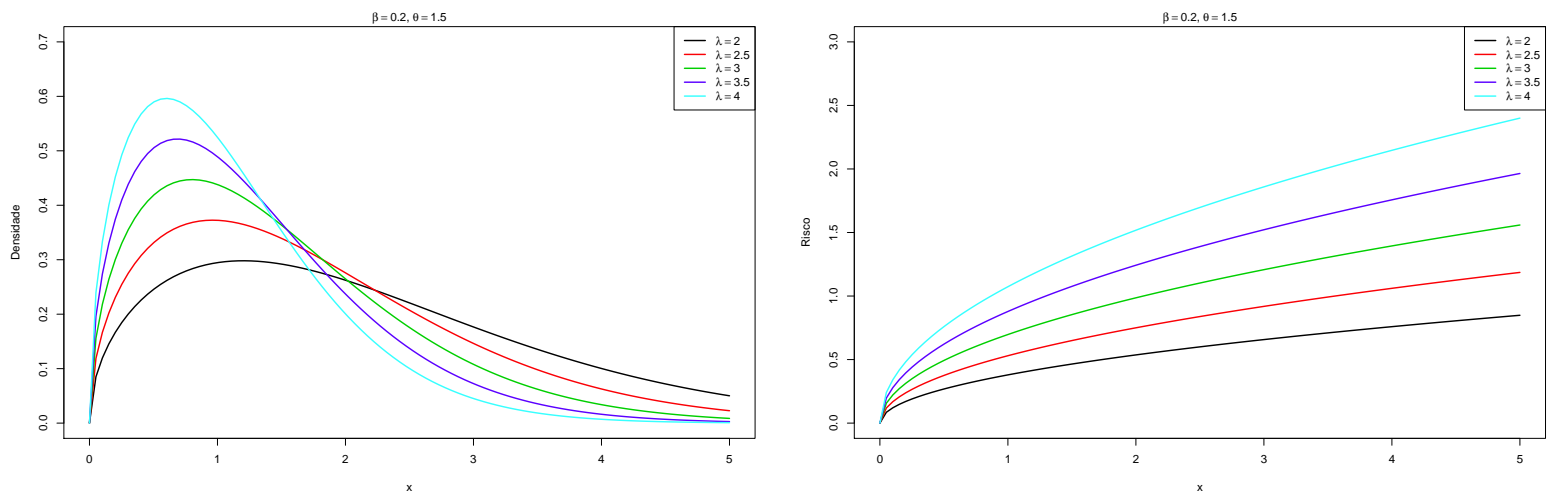

Figura 2: Função densidade de probabilidade e função de risco com $\beta$ e $\theta$ fixos e variando o $\lambda$

A Figura 3 apresenta a função densidade de probabilidade e a função de risco $\operatorname{com} \beta$ e $\lambda$ fixos e variando o $\theta$. Nota-se que a função densidade tem diferentes formas diferenciando de acordo com o $\theta$, quando o $\theta$ aumenta seu formato se torna simétrico. A função de risco é crescente para qualquer valor de $\theta$, no entanto o risco tende a aumentar mais rápido quando o tempo aumenta para valores de $\theta$ maiores que 2 . Um comportamento inverso ocorre para $\theta<2$.
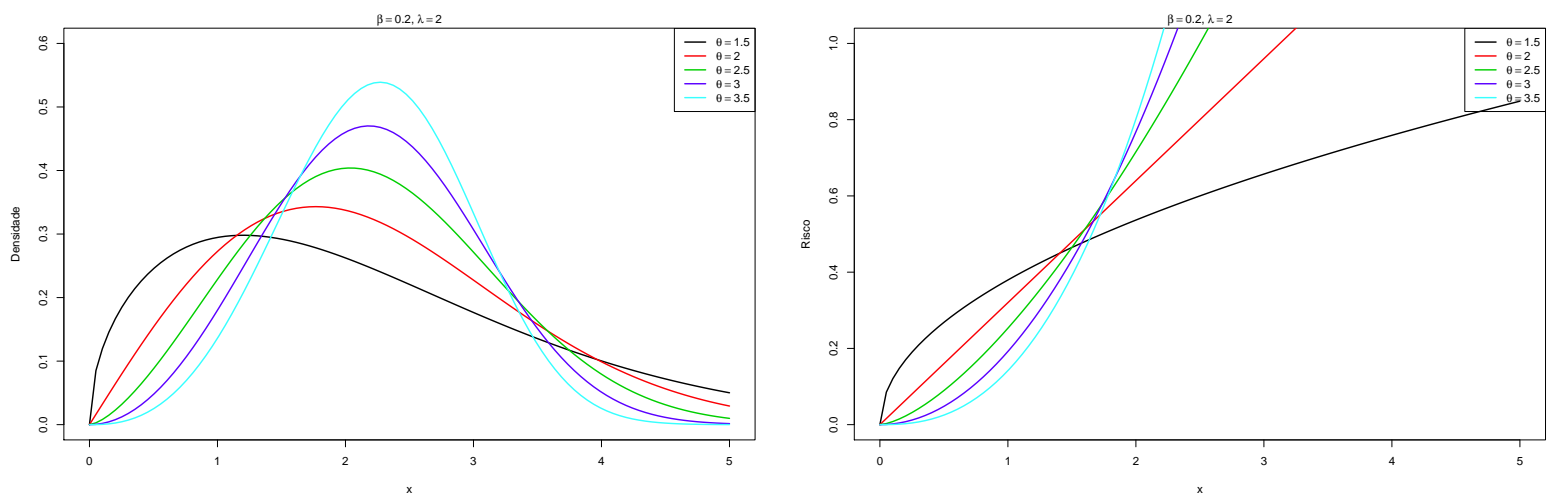

Figura 3: Função densidade de probabilidade e função de risco com $\beta$ e $\lambda$ fixos e variando o $\theta$

A Figura 4 apresenta a média, variância, coeficiente de assimetria e curtose com $\lambda$ e $\theta$ fixos e variando o $\beta$. A média decresce e tende a se concentrar na origem quando se aumenta o $\beta$. A variância decresce quando o $\beta$ cresce.
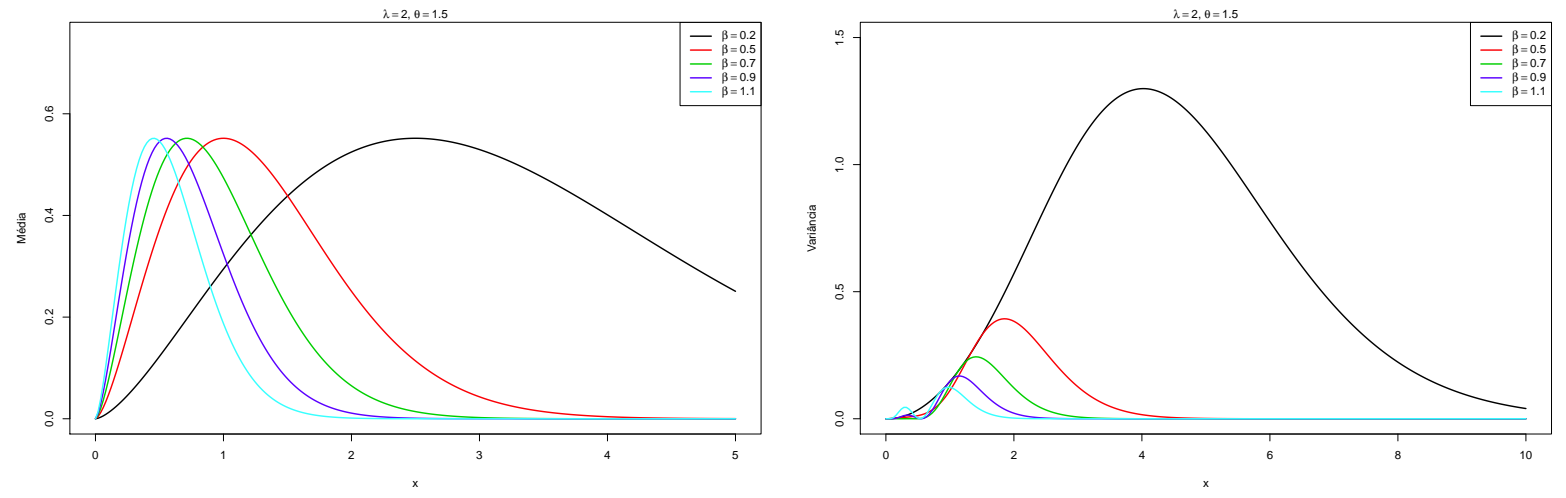

Figura 4: A média e variância com $\lambda$ e $\theta$ fixos e variando o $\beta$ 
A Figura 5 apresenta os coeficientes de assimetria e curtose $\operatorname{com} \lambda$ e $\theta$ fixos e variando o $\beta$. $\mathrm{O}$ coeficiente de assimetria e curtose apresentam resultados semelhantes, para um valor fixo de $\mathrm{x}$, quando o $\beta$ cresce a assimetria e a curtose diminuem e tendem a se concentrar na origem.
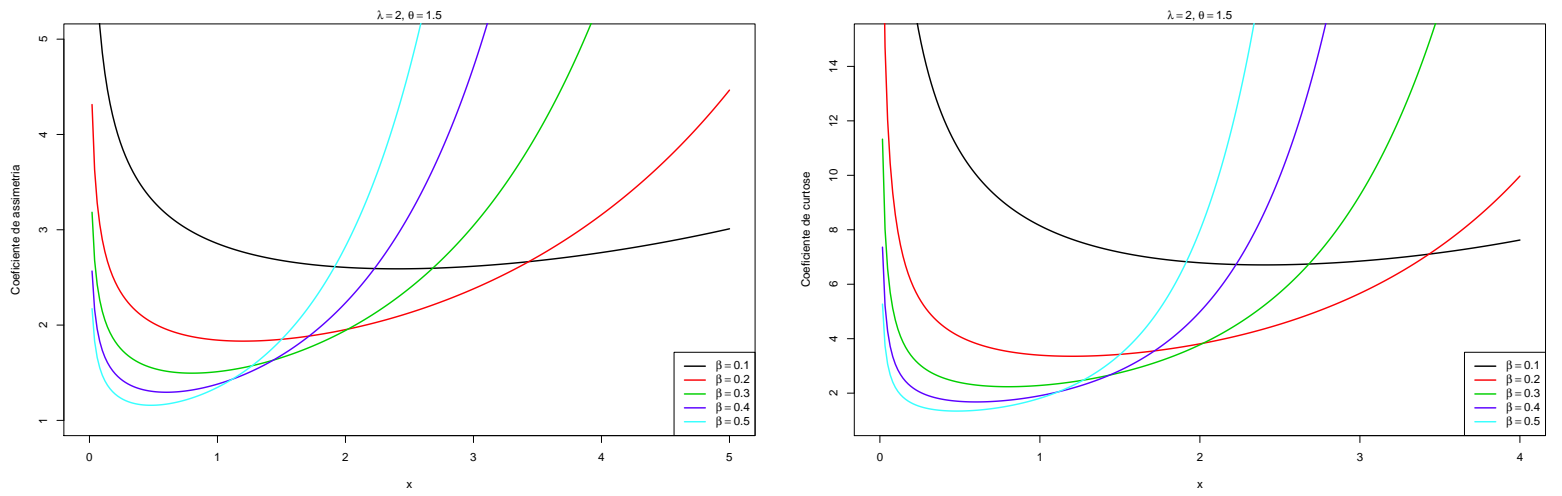

Figura 5: Coeficiente de assimetria e o coeficiente de curtose $\operatorname{com} \lambda$ e $\theta$ fixos e variando o $\beta$

Fez-se uma simulação com 100 valores da distribuição WEZ $\operatorname{com} \beta=1,2, \lambda=2$ e $\theta=1,5$. Ajustou-se aos dados a densidade com os parâmetros verdadeiros acima e com sua estimativa de máxima verossimilhança via algoritmo de estimação BFGS. Os estimadores de máxima verossimilhança foram $\beta=1,56, \lambda=1,56$ e $\theta=1,57$. A Figura 6 mostra o histograma com o ajuste dos parâmetros e da função de log-verossimilhança da distribuição Weibull Exponencial de Zografos.

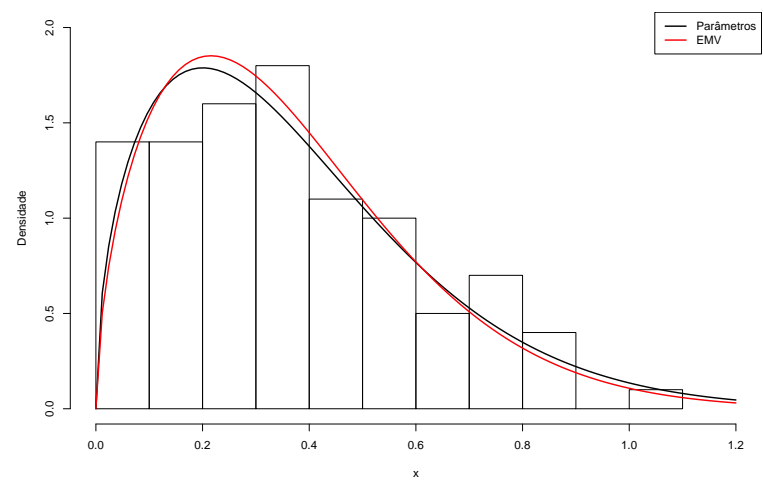

Figura 6: Histograma com ajuste dos parâmetros e da função de log-verossimilhança

Verifica-se que o ajuste das estimativas pelo método de máxima verossimilhança foi melhor do que dos parâmetros estipulados. Para medir qual o melhor ajuste pode-se utilizar a logverossimilhança que é dada por:

$$
l(\boldsymbol{\xi})=\sum_{i=1}^{n} \log f\left(\boldsymbol{\xi} ; x_{i}\right)
$$

O ajuste que obter a maior log-verossimilhança é melhor. Os valores da log-verossimilhança foram 5,79 para os parâmetros estipulados e 5,82 para o ajuste pelo método de máxima verossimilhança. Dessa forma o melhor ajuste foi da estimativa pelo método de máxima verosimilhança. Com isso, conclui-se que a distribuição Weibull Exponencial de Zografos pode ser utilizada para ajustar dados de análise de sobrevivência. 


\section{Conclusões}

Neste artigo, foi introduzida a distribuição Weibull Exponencial de Zografos que é uma generalização da distribuição Weibull pelo método introduzido por [3]. Encontrou-se os quatro primeiros momentos: média, variância, coeficiente de assimetria e curtose. Utilizou-se o método da máxima verossimilhança para ajustar os parâmetros da distribuição Weibull Exponencial de Zografos. Verificando pela log-verossimilhança que as estimativas pelo método de máxima verossimilhança teve melhor ajuste. Concluindo que a distribuição Weibull Exponencial de Zografos pode ser utilizada para ajustar dados de análise de sobrevivência.

\section{Referências}

[1] H. Pham, C-D. Lai. On Recent Generalizations of the Weibull Distribution, IEEE Transactions on reliability, vol. 56, no. 3, 2007.

[2] G. O. Silva, E. M. Ortega, G. M. Cordeiro. The beta modified Weibull distribution. Lifetime Data Anal. 3, 409-30, 2003.

[3] K. Zografos, N. Balakrishnan. On families of beta- and generalized gamma-generated distributions and associated inference. Statistical Methodology. 6, 344-362, 2009.

[4] W. Weibull. A Statistical Distribution Function of Wide Aplicability. ASME Journal of Apliec Mechanics, v. 18, pp. 293-297, 1951. 